\title{
Diabetes Prediction and Analysis using Machine Learning Methods
}

\author{
Sruthi M.S, Sushmitha Magudeswaren, Soniya Tamilarasu, Sushmitha Muralitharen.
}

\begin{abstract}
Different computational procedures and gadgets are open for data examination. At the present time, took the advantages of those open developments to improve the adequacy of the estimate model for the desire for a Type-2 Diabetic Patient. We intend to inquire about how diabetes scenes are impacted by patients' characteristics and estimations. The capable gauge model is required for clinical researchers. Until generally, Type II diabetes was evaluated uncommon in children. The contamination is, nonetheless, creating among youths in peoples with high paces of Type II diabetes in adults. This work presents the adequacy of Gradient Boosted Classifier which is obscure in past current works. It is related to two AI figuring's, for instance, Neural Networks, Random Forest. These estimations are applied to the Pima Indians Diabetes Database (PIDD) which is sourced from the UCI AI storage facility. The models made are surveyed by standard techniques, for instance, AUC, Recall, and Accuracy. As obvious, Gradient helped classifier clobbers other two classifiers in all introduction qualities.
\end{abstract}

Keywords: Catchphrases: Diabetic Patients, Neural Networks, Random Forest, Accuracy.

\section{INTRODUCTION}

Diabetes mellitus is considered as one of the deadliest and relentless ailments for people which causes a development in glucose. If diabetes remains untreated and unidentified, by then various bothers will happen to individuals. This perceiving technique achieves visiting a patient to a demonstrative spot and directing expert, anyway with the help of AI approaches deals with this essential issue in a surged manner. The essential objective of this assessment is to structure a model which can anticipate the likelihood of diabetes in patients with most noteworthy precision. Right now, presently following three AI gathering techniques to be explicit Decision Tree, SVM and Naive Bayes are used to distinguish diabetes at a starting period. These computations are performed on the Pima Indians Diabetes Database (PIDD) which is sourced from the UCI AI store. The shows of all of the three computations are surveyed on various assessments like Precision, Accuracy, F-Measure, Recall, etc.

Revised Manuscript Received on April 30, 2020.

* Correspondence Author

Sruthi M S*, Assistant Professor in the department of Computer Science and Engineering at Sri Krishna College of Technology.

Sushmitha Magudeswaren, Student of B.E in Computer Science and

Soniya Tamilarasu, Student of B.E in Computer Science and Engineering at Sri Krishna College of Technology, Coimbatore.

Sushmitha Muralitharen ,Student of B.E in Computer Science and Engineering at Sri Krishna College of Technology, Coimbatore.

(C) The Authors. Published by Blue Eyes Intelligence Engineering and Sciences Publication (BEIESP). This is an open access article under the CC BY-NC-ND license (http://creativecommons.org/licenses/by-nc-nd/4.0/) Engineering at Sri Krishna College of Technology, Coimbatore.

Request systems are broadly used in the clinical field for gathering data into different classes as demonstrated by some obliges moderately an individual classifier. Diabetes mellitus is an affliction which impacts the limit of the body in making the hormone insulin, which in this way makes the assimilation of sugar odd and raise the degrees of glucose in the blood. In Diabetes an individual can encounter the evil impacts of high glucose. Increment thirst, heighten hunger, and Frequent pee these are completely considered as certain reactions caused on account of high glucose. Various burdens occur if diabetes remains untreated, it achieves some outrageous entrapments consolidate diabetic ketoacidosis(DKA) and Hyperosmolar Hyperglycemic Nonketotic Syndrome (HHNS). Diabetes is assessed as a principal authentic prosperity matter during which the extent of sugar substance can't be controlled. Diabetes isn't simply impacted by various segments like stature, weight, acquired factor and insulin yet the huge clarification considered is sugar center among all components.

\section{RELATED WORK}

Sajida et al. in [20] discusses the activity of Ada lift and Bagging gathering AI systems [18] usingJ48 decision tree as the explanation behind gathering the Diabetes Mellitus and patients as diabetic or non-diabetic, considering diabetes chance segments. The tests were done and the results show that Ada help AI troupe methodology outmaneuvers well moderately pressing similarly as a J48 decision tree.

Rabi et al. in [19] organized a structure for diabetes gauge, whose basic point is to foresee diabetes a contender is suffering at a particular age. The proposed system is organized subject to the possibility of AI, by applying the decision tree. The results got were gone to the great as the organized system works splendidly in envisioning the diabetes scenes at a particular age, with higher precision using Decision tree[22], [7].

Pradhan et al. in [4] used Genetic programming (GP) for the readiness and testing of the database for the desire for diabetes by using the Diabetes enlightening assortment which is sourced from the UCI storage facility. The cultivated results using Genetic Programming [25], [21] gives perfect precision when appeared differently in relation to other realized methodologies. There can be in a general sense improved blunder by saving less exertion for classifier age. It demonstrates reality to be significant for diabetes figure easily. Rashid et al. in [28] organized a figure model with two sub-modules to anticipate diabetes disease. In the essential module ANN (Artificial Neural Network) is used and in the second module FBS (Fasting Blood Sugar) is used. 
Decision Tree (DT)[10] is used to recognize the reactions of diabetes in patients prosperity.

Nongyao et al. in [17] applied an estimation which is used to orchestrate the peril of diabetes mellitus. To fulfill the objective maker has applied the going with AI portrayal methodologies to be explicit Decision Tree, Artificial Neural Networks, Logistic Regression and Naive Bayes. For improving the intensity of organized model Bagging and Boosting techniques are used. Experimentation results show the Random Forest estimation gives perfect results among all the computations used.

\section{PHILOSOPHYAND MODEL DIAGRAM}

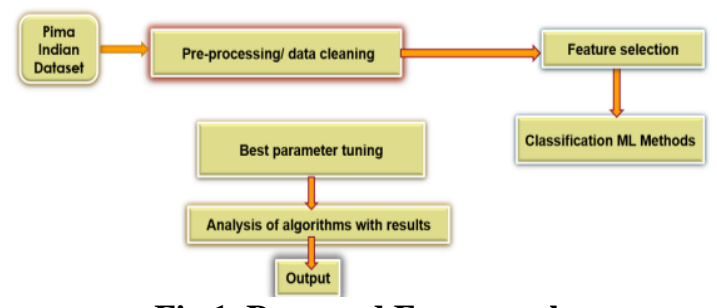

Fig.1. Proposed Framework

\section{Dataset Used:}

This dataset is at first from the (NIDDKD) National Institute of Diabetes and Digestive and Kidney Diseases. The objective of the dataset is to envision logically whether a patient is affected by diabetes or not, established on certain symptomatic estimations associated with the dataset. There are a couple of goals were determined to the assurance of these events from a greater database. From the dataset, all patients here are females at the hour of at any rate 21 years old of Pima Indian inheritance. Right now, picked datasets involve a couple of clinical pointer components and one target variable, Outcome. Pointer factors consolidate pregnancies, BMI, insulin level, age, and so on.

\begin{tabular}{|c|c|}
\hline Content & attribute Description \\
\hline Pregnancies & Number of times pregnant \\
\hline Glucose & $\begin{array}{l}\text { Plasma glucose fixation } 2 \text { hours in an } \\
\text { oral glucose resistance test }\end{array}$ \\
\hline Blood pressure & Diastolic circulatory strain ( $\mathrm{mm} \mathrm{hg}$ ) \\
\hline Skin thickness & Triceps skin overlap thickness (mm) \\
\hline Insulin & Hour serum insulin $(\mathrm{mu} \mathrm{u} / \mathrm{ml})$ \\
\hline BMI & $\begin{array}{l}\text { Body mass file (weight in } \mathrm{kg} / \text { (tallness } \\
\text { in } \mathrm{m})^{\wedge} 2 \text { ) }\end{array}$ \\
\hline $\begin{array}{l}\text { Diabetes family } \\
\text { function }\end{array}$ & Diabetes family work \\
\hline Age & Age (years) \\
\hline Outcome & $\begin{array}{l}\text { Class variable (0 or } 1) 268 \text { of } 768 \text { are } \\
1 \text {, the others are } 0\end{array}$ \\
\hline
\end{tabular}

Fig.2.Description of Variables

\section{Ai Methods Used:}

\section{A.Neural Networks:}

A neural system is a kind of AI arrangement model calculation that tries to perceive basic connections in a lot of information through a procedure that imitates the manner in which the human cerebrum works. Right now arranges allude to the frameworks of neurons, either natural or counterfeit in nature. The information signal from the outside world is gotten as an example and picture as a vector in the Artificial Neural Networks. Every one of the sources of info is then increased by it's relating loads (these loads are the subtleties utilized by the fake neural systems technique to take care of a specific issue). Today, numerous business issues, for example, deals estimating, client explore, information approval, and hazard the board are comprehended utilizing the neural system technique. For instance, at Stetson we apply neural systems for time-arrangement forecasts, abnormality identification in information, and common language understanding. The neural philosophy can learn, review and sum up from the given information by reasonable task and change of loads. The aggregate conduct of the neurons portrays its computational force, and no single neuron conveys explicit data. The benefit of ANNs is one that ,it can learn and demonstrate non-straight and complex connections, which is extremely significant in light of the fact that, all things considered, a large number of the connections among sources of info and yields are non-direct and furthermore a perplexing one.

\section{B . Random Forest:}

In an AI the Random Forests calculation is a learning model for order, relapse thus on,that can be worked well. Individual choice trees that can work as an outfit. A model's forecast depends on an individual tree in the irregular timberland that lets out a class expectation and the class with the most votes . The essential idea driving arbitrary choice woodland technique is a basic however ground-breaking one - the shrewdness of groups. In present day part of information science talk, the explanation that the arbitrary backwoods model works are well. The least connection between's models is the key. Much the same as how ventures with low relationships (like stocks and securities) meet up to frame a portfolio which is more noteworthy than that of its whole of parts, uncorrelated models can deliver gathering expectations which is more exact than that of its individual forecasts. The principle purpose behind this impact is the trees shield each other from their individual mistakes (as long as they don't continually all blunder in the equivalent direction).Some of the trees might not be right, While numerous different trees will be correct, so as a gathering the trees can move in the right course.

\section{Gradient Boosting:}

The AI calculation Gradient Boosting is utilized for finding out about relapse and order issues, thus, the calculation delivers a forecast model as a powerless expectation models which is normally known as the choice trees. The calculation can be the most effectively clarified by first presenting the calculation called AdaBoost. 
The AdaBoost Algorithm can be characterized as,it starts via preparing a choice tree . Right now perception is allocated an equivalent weight.

Angle Boosting strategy trains numerous models in a steady, added substance, and successive way. Inclination boosting works better since it is a vigorous out-of-the-crate classifier (regressor) that can perform on a dataset on which negligible exertion has been spent on cleaning and can learn complex non-direct choice limits through boosting. In the event that you cautiously tune parameters, slope boosting can bring about great execution than irregular timberlands. In any case, inclination boosting may not be a superior decision in the event that you have a ton of clamor, as it can result in over fitting. This calculation likewise will in general be harder to tune than arbitrary timberlands.

\section{RESULTS AND DISCUSSION}

\begin{tabular}{|l|l|l|l|}
\hline CONTENT & $\begin{array}{l}\text { NEURAL } \\
\text { NETWORK }\end{array}$ & RANDOMFOREST & $\begin{array}{l}\text { GRADIENT } \\
\text { BOOSTED } \\
\text { CLASSFIER }\end{array}$ \\
\hline RECALL & 0.701 & 0.656 & 0.761 \\
\hline AUC & 0.907 & 0.907 & 0.942 \\
\hline ACCURACY & 0.838 & 0.822 & 0.897 \\
\hline
\end{tabular}

Fig.3. Examination and Results of Three Classifiers

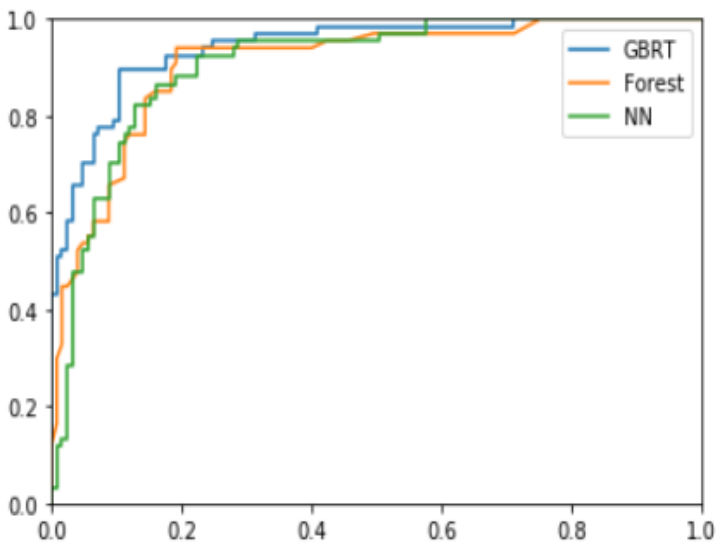

Fig.4. ROC Comparison of Three

\begin{tabular}{|l|l|}
\multicolumn{2}{|c|}{ Classifier } \\
\hline Classifiers & Accuracy \\
\hline RBF SVM & $64 \%$ \\
\hline Direct SVM & $76 \%$ \\
\hline Neural Net & $60 \%$ \\
\hline Choice Tree & $66 \%$ \\
\hline Gaussian Process & $68 \%$ \\
\hline Closest Neighbours & $71 \%$ \\
\hline QDA & $73 \%$ \\
\hline AdaBoost & $72 \%$ \\
\hline Gullible Bayes & $73 \%$ \\
\hline Irregular Forest & $73 \%$ \\
\hline Spearman neural network & $84 \%$ \\
\hline Spearman Random Forest & $82 \%$ \\
\hline Spearman Gradient Boosted & $90 \%$ \\
\hline
\end{tabular}

Fig.5. Examination with other Existing Classifiers

\section{CONCLUTION}

Information Analytics is the system of recover an example from huge informational index regarding AI, information base, and insights. AI is a particularly helpful in choosing the line of treatment to be trailed by separating information and information from such reasonable databases. The similar investigation is performed with the assistance of following execution assessment parameters, for example, exactness, affectability, particularity for assessing the great execution for diabetic expectation.

\section{REFERENCES}

1. Zou, Quan, KaiyangQu, YameiLuo, Dehui Yin, Ying Ju, and Hua Tang. "Predicting diabetes mellitus with machine learning techniques."Frontiers in genetics 9 (2018): 515.

2. Wu, Han, Shengqi Yang, Zhangqin Huang, Jian He, and Xiaoyi Wang. "Type 2 diabetes mellitus prediction model based on data mining."Informatics in Medicine Unlocked 10 (2018): 100-107.

3. A. Géron, (2017). Hands-on machine learning with Scikit-Learn and TensorFlow: concepts, tools, and techniques to build intelligent systems." O'Reilly Media, Inc.".

4. Guo-li DU, et al. "Metabolic Risk Factors of Type 2 Diabetes Mellitus and Correlated GlycemicControl/Complications: A Cross-Sectional Study between Rural and Urban Uygur Residents in Xinjiang Uygur Autonomous Region,"PloS one, vol. 11, 2016.

5. Christian, BOMMER, et al. "The global economic burden of diabetes in adults aged 20-79 years: a cost-of-illness study," The lancet Diabetes \& endocrinology, vol. 5, pp. 423-430, 2017.

6. Alessandra M., et al. MANTOVANI, "Relationship between amputation and risk factors in individuals with diabetes mellitus: a study with Brazilian patients," Diabetes \& Metabolic Syndrome: Clinical Research \& Reviews, vol. 11, pp. 47-50, 2017.

7. ArturoCorbatón ANCHUELO, Rafael Cuervo PINTO, and Manuel Serrano. RÍOS, "La diabetes mellitus tipo 2 comoenfermedad cardiovascular,"RevistaEspañola de CardiologíaSuplementos, vol. 7 , pp. 9A-22A, 2007.

8. Jonathan E. SHAW, Richard A. SICREE, and Paul Z. ZIMMET, "Global estimates of the prevalence of diabetes for 2010 and 2030," Diabetes research and clinical practice, vol. 87, pp. 4-14, 2010.

9. L. M., et al. PEÑA-LONGOBARDO, "Is quality of life different between diabetic and non-diabetic people? The importance of cardiovascular risks,"PloS one, vol. 12, 2017.

10. M. L., ALVA, et al. "The impact of diabetes-related complications on healthcare costs: new results from the UKPDS (UKPDS 84)," Diabetic Medicine, vol. 32, pp. 459-466, 2015.

\section{AUTHORS PROFILE}

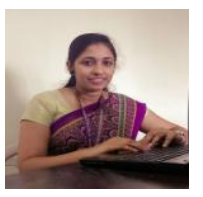

Sruthi M S , working as Assistant Professor in the department of Computer Science and Engineering at Sri Krishna College of Technology. Currently doing her research on Machine Learning.Completed UG in Computer Science and Engineering, PG in CSE specialized in Networks.

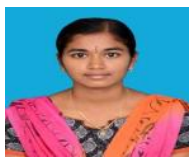

Sushmitha Magudeswaren, is a student of B.E in Computer Science and Engineering at Sri Krishna College of Technology, Coimbatore.

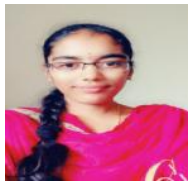

Soniya Tamilarasu, is a student of B.E in Computer Science and Engineering at Sri Krishna College of Technology, Coimbatore.

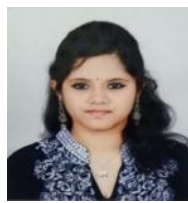

Sushmitha Muralitharen , is a student of B.E in Computer Science and Engineering at Sri Krishna College of Technology, Coimbatore. 